\title{
Evaluación de características microscópicas de semen de llama (Lama glama) crioconservados en dos dilutores
}

\section{Microscopic evaluation of semen characteristics llama (Lama glama) cryopreserved two dilutors}

\author{
Laruta-Limachi Felicidad ${ }^{1,2^{*}}$, Loza-Murguia Manuel Gregorio ${ }^{3.4}$, Delgado-Callisaya Pedro Ángel ${ }^{1,2}$,
}

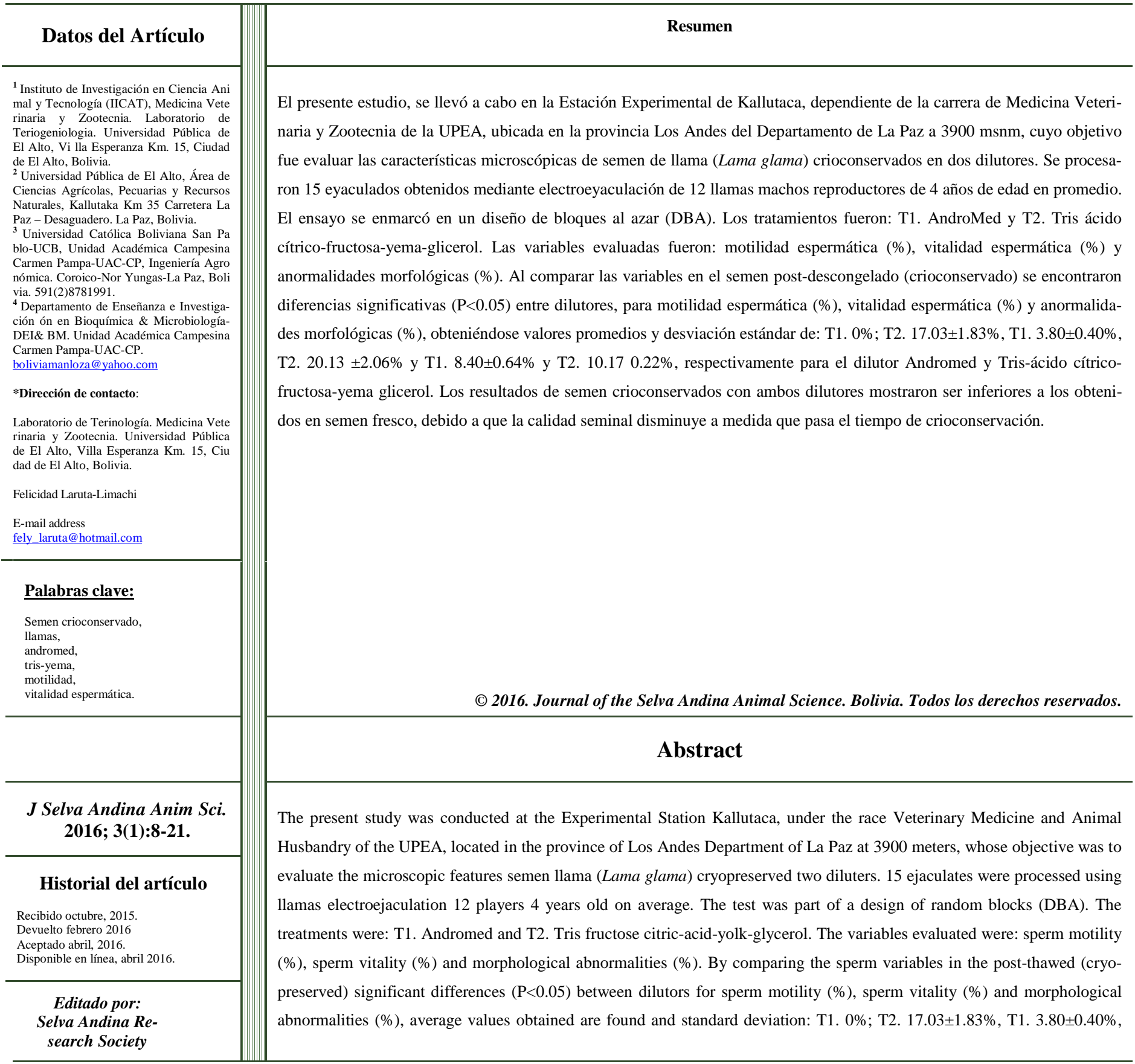




\begin{tabular}{|c|c|}
\hline $\begin{array}{l}\text { Key words: } \\
\text { Cryopreserved semen, } \\
\text { flames, } \\
\text { andromed, } \\
\text { tris-egg, } \\
\text { sperm motility, } \\
\text { vitality. }\end{array}$ & $\begin{array}{l}\text { T2. } 20.13 \pm 2.06 \% \text { and T1. } 8.40 \pm 0.64 \% \text { and T2. } 10.17 \pm 0.22 \% \text { respectively for the dilutor Andromed Tris-citric acid and } \\
\text { fructose-yolk-glycerol. The results of cryopreserved semen diluters both proved to be lower than those obtained in fresh } \\
\text { semen, because the semen quality decreases as time passes cryopreservation. } \\
\text { C 2016. Journal of the Selva Andina Animal Science. Bolivia. All rights reserved. }\end{array}$ \\
\hline
\end{tabular}

\section{Introducción}

La crianza de alpacas y llamas constituye una actividad económica de vital importancia para un amplio sector de la población altoandina de Perú, Bolivia, Argentina, Chile y Ecuador. Se estima que alrededor de 500 mil familias campesinas de la región andina dependen directamente de la actividad con camélidos sudamericanos (Ruiz 2005), otras se benefician aprovechando su subproducto como fibra, carne, piel y estiércol, además la llama por su mayor tamaño y fortaleza es utilizada como animal de transporte (Cárdenas et al. 1999).

Una mayor área de la región Alto Andina de Bolivia, es apta para el repoblamiento de llamas (Ratto et al. 1999), sin embargo el crecimiento vegetativo de esta especie es de 6\% anual (Bravo et al. 2002), porcentajes de fertilidad debajo de $50 \%$, han sido reportados por diferente autores, en sistemas de empadre, que no garantizan el inicio de repoblamiento.

Los primeros trabajos de colección de semen en camélidos sudamericanos (CSA) se hicieron con el uso de fundas vaginales (Mogrovejo 1952), luego se utilizó electroeyaculación (Fernández-Baca \& Calderón 1965), actualmente se emplea vagina artificial con cérvix simulada, en un maniquí en posición copulatoria (Sumar \& Leyva 1981). La vagina artificial tiene que ser mantenida a temperatura corporal de la hembra con el apoyo de una frazadilla eléctrica (Bravo et al. 1997a) para permitir que el macho no interrumpa la cópula.
El conocimiento de la biología reproductiva de los camélidos sudamericanos es aún insuficiente, especialmente en lo referido a la fisiología reproductiva del macho. La inseminación artificial es una de las tecnologías que ha contribuido al progreso genético en diversas especies de animales de producción, especialmente en el bovino, sin embargo, existe muy poca información sobre la colección, características, evaluación y conservación del semen en la alpaca, debido a la falta de una metodología confiable y reproducible para la colección de semen (Ferré $\&$ Werkmeister 1996). Las principales limitaciones son la colección de semen, la falta de conocimiento sobre su composición, viscosidad y uso de dilutores (Bustinza 2001).

El semen de la alpaca es altamente viscoso, lo que hace difícil su manejo en el laboratorio.

La dificultad en la colección del semen es atribuido entre otras causas, a las características peculiares de monta (posición de cúbito ventral), que dura entre 20 a 30 min, aproximadamente. Además los animales son de temperamento nervioso, lo que dificulta su manejo (Fernández-Baca \& Calderón 1965).

La conservación de las células espermáticas se basa, esencialmente, en el uso de dilutores que proporcionen los nutrientes que neutralicen los cambios de pH producido por el metabolismo de los azúcares (efecto tampón), que protejan a los espermatozoides del descenso de temperatura durante el proceso de congelación (Herrera 1986). 
A la fecha, se reporta el uso de varios dilutores para semen de alpacas, entre los que se encuentran la leche descremada, fosfato salino tamponado, glucosa-citrato, yema de huevo-glucosa-citrato, tryladil y tris tamponado; aunque parece que el mejor dilutor para semen de alpacas es el tris tamponado (Bravo 1989), existen resultados contradictorios y muchas veces no repetibles. El presente estudio tuvo como objetivo estudiar el efecto de tres dilutores en la conservación del semen de alpacas.

Con este trabajo de investigación se pretende conservar, mantener fértiles y motiles a los espermatozoides el mayor tiempo posible, planteándonos el objetivo, evaluar la motilidad, vitalidad y anormalidades espermáticas del semen de llama crioconservado en dos dilutores (AndroMed y Tris-ácido cítrico-fructosa-yema-glicerol).

\section{Materiales y métodos}

Localización de la investigación. El presente trabajo de investigación se realizó en instalaciones de la Estación Experimental de Kallutaca, Laboratorio de Teriogeniología de Medicina Veterinaria y Zootecnia, Universidad Pública de El Alto (UPEA), localizado en la provincia Los Andes del Departamento de La Paz a una distancia de $27 \mathrm{Km}$ de la ciudad, geográficamente se encuentra ubicada a $16^{\circ} 31^{\prime} 28^{\prime \prime}$ de latitud sur y $68^{\circ} 20^{\prime} 39^{\prime \prime}$ de longitud oeste y una altitud de 3990 msnm (IGM 2005).

Se utilizó 12 llamas machos tipo Q'ara (Marín et al. 2008) de 4 años de edad, procedentes de la localidad de Palcoco-Provincia Los Andes del Departamento de La Paz.

La selección de los machos fue previa evaluación de dentadura para estimar su edad (técnica de boqueo), y en base a la condición corporal, su estado sanitario, tamaño, forma, consistencia y elasticidad de los testículos y epidídimo, así como las condiciones del prepucio y el pene.

Figura 1 Área de estudio Kallutaca, departamento de La Paz, Bolivia

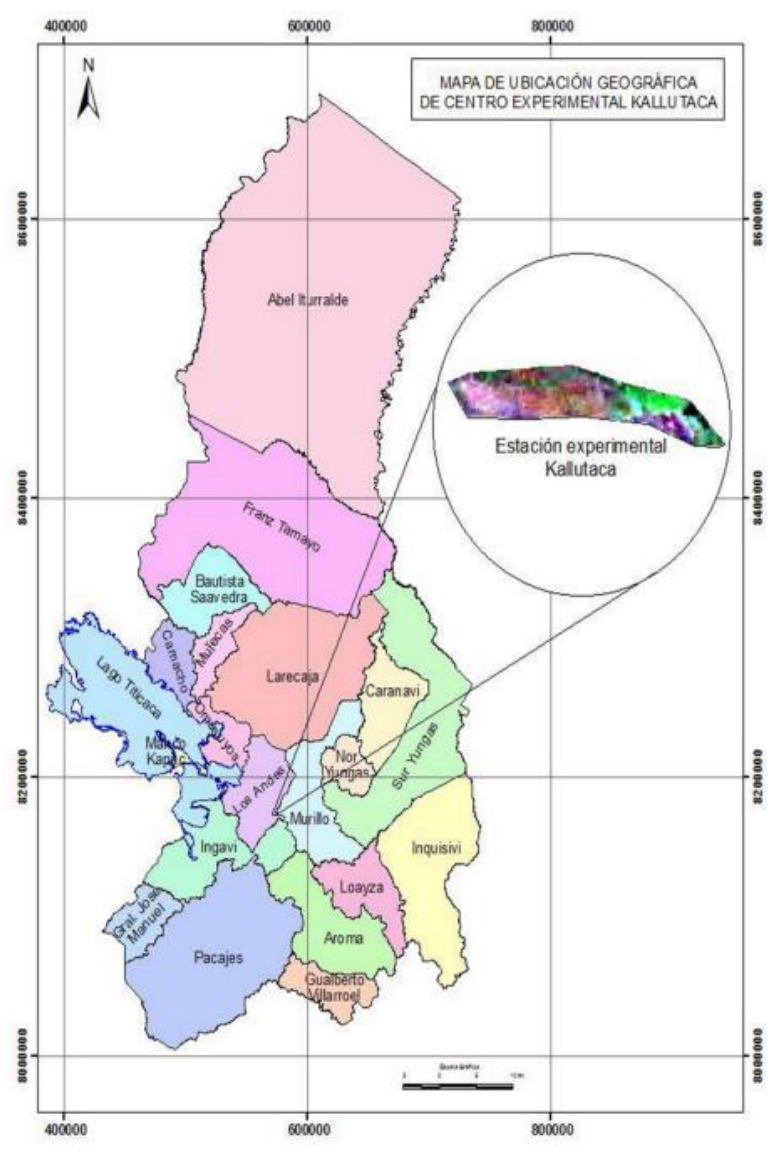

Se los separo en tres grupos de 4 individuos, el primer grupo constaba de llamas de 3 años con un peso promedio de $70 \mathrm{Kg}$, el segundo grupo de 4 años con peso de $80 \mathrm{~kg}$, y el tercer mayor a cinco años con $100 \mathrm{~kg}$, de peso vivo. Cada grupo estaba separado en un corral de $18 \mathrm{~m}^{2}$, se los adaptó a las condiciones del lugar de estudio. Todas las llamas fueron alimentadas con tres dietas: 1) heno de Alfalfa $(40 \%)+$ heno de Pasto Ovillo (60\%) 2) heno de Avena (100\%) 3) heno de Cebada (100\%).

Colecta de semen. La colecta del semen se realizó dos veces por semana obteniendo un volumen un promedio de $1.5 \mathrm{~mL}$ por individuo, en su colecta se 
aplicó Xilazina al $2 \%$ en una dosis de $0.2 \mathrm{mg} / \mathrm{kg}$ peso vivo (PV), intra muscular (IM) y Ketamina al $10 \%$ en una dosis de $4 \mathrm{mg} / \mathrm{kg}$ PV (IM) (Giuliano et al. 2008), con el fin de que el animal no pueda reaccionar durante el proceso de electroeyaculación (EE), cuyo efecto empezaba entre 6-8 min después de la administración por IM, con una duración total de 30 a 45 min.

La electroeyaculación (EE) se realizó con un Electroeyaculador de camélido adaptado a un pulsador de bovino (LANE MANUFACTURINGPULSATOR IV) alimentada por energía eléctrica y una mesa de colección de semen para camélidos, la posición del animal fue de decúbito ventral. La mesa presentó las siguientes medidas: $1.20 \mathrm{~m}$ por 0.80 $\mathrm{m}$, el cual tenía un orificio rectangular de $0.3 \mathrm{~m}$ por $0.2 \mathrm{~m}$ para direccionar el pene al tubo colector durante la EE, Cruz \& Delgado (2011).

Para la EE fue utilizado un electrodo rectal bipolar, con tres tiras longitudinales de cobre de $9 \mathrm{~cm}$ de largo, las tiras de cobre presentaban $0.4 \mathrm{~cm}$ de distancia entre ellas, fueron construidas de forma que estuviesen salidas en aproximadamente $0.2 \mathrm{~cm}$ el diámetro del transductor rectal fue de $2.0 \mathrm{~cm}$ de ancho.

El transductor rectal fue debidamente lubricado con vaselina liquida e introducido en el recto de 10 a 15 $\mathrm{cm}$ de acuerdo al animal, las tiras longitudinales posicionadas ventralmente, ejerciendo una ligera presión en la pared rectal, con el objetivo de aumentar el contacto con la región del plexo nervioso pélvico.

Los electrochoques fueron realizados en una serie de: 15 estímulos de $3 \mathrm{~V}, 20$ estímulos de $4 \mathrm{~V}$ y 25 estímulos de $6 \mathrm{~V}$ con intervalos de 5 segundos de descanso entre cada pulsación, haciendo un total de 60 estímulos por serie. La generación de los estímulos fue realizada girando lentamente el control del pulsador bovino hasta llegar al voltaje deseado, el 11 cual fue mantenido de 15 a $25 \mathrm{~s}$, y enseguida retornado rápidamente a $0 \mathrm{~V}$ (apagado). La duración de los estímulos fue alrededor de 8-10 min.

Preparación de los dilutores. Se realizó con asepsia, de acuerdo a lo recomendado Delgado-Callisaya (2010). Antes de la colecta de semen se realizó la preparación de dilutores (AndroMed o Tris-ácido cítrico-fructosa-yema-glicerol), luego se realizó la colecta de semen, protocolo para la congelación del semen y evaluación de características microscópicas de semen post - congelamiento

Tabla 1 Componentes para la preparación de $50 \mathrm{~mL}$ de dilutor TRIS, usado para la conservación de semen de llamas (Lama glama)

\begin{tabular}{lc}
\hline Insumo & Cantidad \\
\hline TRIS & $1.817 \mathrm{~g}$ \\
Ácido cítrico & $0.995 \mathrm{~g}$ \\
Fructosa & $0.550 \mathrm{~g}$ \\
Gentamicina & $120 \mu \mathrm{g} / \mathrm{mL}$ \\
Yema de huevo & $20 \%$ \\
\hline
\end{tabular}

AndroMed® contiene fosfolípidos, TRIS, ácido cítrico, azúcares, antioxidantes, tampones, glicerina, agua de altísima pureza y antibióticos, de acuerdo a la Directiva de la UE 88/407 (Tilosina, Gentamicina, Espectinomicina, Lincomicina.

Para disolver la viscosidad del semen de llama se utilizó extracto de jugo de piña en una relación de 1:0.5 (semen: extracto de jugo de piña), antes de evaluar las características microscópicas del semen de llama. El congelamiento se realizó colocando las pajuelas a $4 \mathrm{~cm}$ sobre el nivel del nitrógeno líquido por 15 min. Por último se sumergió las pajuelas al nitrógeno líquido. Para la determinación de la motilidad espermática antes y después de ser congelados se utilizó el analizador de semen digital (ISAS CASA). Para la determinación de espermatozoides vivos y muertos se empleó la técnica descrita por Santalla (2013), haciendo el uso de la tinción de 
Eosina-Nigrosina, para la determinación espermatozoides anormales, se utilizó los mismos frotis preparados para el porcentaje de vitalidad. Para diferenciar las anormalidades primarias y secundarias, se utilizó como referencia las anormalidades descritas por (Hafez 1996, López 2001, Valle 2013) en Camélidos Sudamericanos.

Evaluación de características macroscópicas del semen fresco (pre congelado).

Volumen. El volumen del eyaculado se determinó por lectura directa, registrándose el volumen colectado en el tubo Falcón (mL).

Color. La evaluación del color, se realizó por observación directa, en el tubo colector (Falcón), considerando para tal efecto tres tonalidades: cristalino, gris y lechoso, de acuerdo a Bustinza (2001).

Evaluación de características microscópicas del semen pre y post descongelado.

Concentración. Esta evaluación solamente se hizo para el semen fresco y se realizó mediante el sistema ISAS CASA utilizando para esto el módulo de concentración.

Motilidad espermática. Para la determinación de la motilidad espermática pre y post descongelación, se utilizó el analizador de semen digital (ISAS CASA), una micropipeta de 10-100 $\mu \mathrm{L}$, un portaobjetos y un cubreobjetos. El portaobjetos y cubreobjetos fueron calentados en la placa térmica a una temperatura de $38{ }^{\circ} \mathrm{C}$, luego se colocó $10 \mu \mathrm{L}$ de semen diluido con jugo de piña en el portaobjetos y luego se cubrió con una lámina cubreobjetos, la lámina preparada fue llevada al microscopio del equipo ISAS CASA, para su evaluación en un lente de 10x, tomándose 3 puntos de referencia, para determinar el porcentaje de espermatozoides que presentan un movimiento rectilíneo y progresivo (correcto), descartando de esta forma aquellos que presentan un movimiento circular (anormal), esto según Delgado-Callisaya (2010).
Porcentaje de espermatozoides vivos y muertos. Para la determinación de espermatozoides vivos y muertos pre y post-descongelación se empleó la técnica descrita por Evans \& Maxwell (1987), haciendo el uso de la tinción de Eosina-Nigrosina, para lo cual se usó un portaobjeto templado a $37{ }^{\circ} \mathrm{C}$ aproximadamente, otro portaobjeto para realizar el frotis y un microscopio, procediéndose de la siguiente manera: i) en el extremo del portaobjeto, se colocó $10 \mu \mathrm{L}$ de semen puro, y $10 \mu \mathrm{L}$ de Eosina al 2 $\%+10 \mu \mathrm{L}$ Nigrosina al 1\%, se homogenizó, ii) se procedió a extender sobre el portaobjetos, de tal manera que se formó una delgada película sobre el portaobjetos, iii) se dejó secar la muestra, y se procedió a observar al microscopio con aumento de 40x, iv) contando 100 células. Del número de células contadas se determinó los espermatozoides vivos y muertos en porcentaje.

Para diferenciar los vivos de los muertos con la coloración de la Eosina-Nigrosina, se tomaron en cuenta los resultados obtenidos por diferentes autores, los que señalan que los espermatozoides muertos a diferencia de los vivos se tiñen con la Eosina (color rojo) en especial sus cabezas (por permeabilidad celular), mientras que los vivos permanecen incoloros según se aprecia contra el fondo oscuro de Nigrosina (Torres 1989, Agraz 1989).

La determinación dada en porcentaje se estimó con la siguiente fórmula Garabito (2003).

$$
\% \mathbf{E V}=\frac{\mathrm{N}^{\circ} \text { Epz. Vivos }}{\mathrm{N}^{\circ} \text { Epz. Contados (Vivos + Muertos) }} \times 100 \%
$$

$\% \mathbf{E V}=$ Porcentaje de espermatozoides vivos $\mathbf{E p z}=$ Espermatozoides

Porcentaje de espermatozoides anormales. Se contaron todos los espermatozoides en los cuatro campos microscópicos del frotis, enumerándolos, como formas anormales.

Para diferenciar las anormalidades primarias y secundarias, se utilizó como referencia las anormali- 
dades descritas por (Hafez 1996, López 2001, Valle 2013) en Camélidos Sudamericanos.

Anormalidades primarias. Cabeza gigante, microcéfalo, colas enrolladas, colas rotas, cabezas dobles, doble cola, colas torcidas alrededor de la cabeza de los espermatozoides.

Anormalidades secundarias. Cabeza suelta o sola, cola sola, colas en gancho, cola doblada, su determinación en porcentaje, se estimó según la fórmula, Mendoza (2001).

\% EPZ. ANORM $=\frac{\mathrm{N}^{\circ} \text { Epz. Anormales }}{\mathrm{N}^{\circ} \text { Epz. Contados }} \times 100 \%$

\% EPZ. ANORM = Porcentaje de espermatozoides anormales

Se aplicó el Diseño de Bloques Completamente al Azar (DBCA), y la prueba de comparación de medias Duncan a un nivel de significancia de $5 \%$.

Modelo estadístico

Se aplicó el Diseño de Bloques Completamente al Azar (DBCA). Cuyo modelo estadístico propuesto por Ochoa (2007) es el siguiente:

$$
X \mathbf{i j}=\mu+\alpha \mathbf{i}+\beta \mathbf{j}+\varepsilon \mathbf{i j}
$$

$\mathbf{X}_{\mathrm{ij}}=$ Una observación cualquiera

$\mu=$ Media genera

$\boldsymbol{\alpha}_{\mathrm{i}}=$ Efecto del i-ésimo dilutor (AndroMed y Tris-ácido cítrico-fructosa-yema-glicerol)

$\boldsymbol{\beta}_{\mathbf{j}}=$ Efecto del j-ésimo bloque (Número de llama)

Evaluación de características macroscópicas y microscópicas de semen fresco

\section{Resultados}

Análisis estadístico.

Prueba de Duncan. Al encontrar diferencias significativas al ANVA para DBCA se utilizó la prueba de comparación de medias Duncan a un nivel de significancia de $5 \%$.

Variables. Factores de estudio. Se trabajó con un solo factor, siendo el factor de estudio dilutores:

Dilutor 1 = AndroMed (A), Dilutor 2 = Tris-ácido cítrico-fructosa-yema-glicerol ( $\mathrm{T}$ - Y)

Variables de respuesta. Las variables de respuesta fueron agrupadas en tres grupos:

Grupo 1 (variables macroscópicas y microscópicas de semen fresco)

Volumen, Color, Concentración espermática (millones/mL), Motilidad espermática (\%), Vitalidad espermática $(\%)$

Grupo 2 (variables microscópicas de semen post descongelado)

Motilidad espermática (\%), Vitalidad espermática $(\%)$, Espermatozoides anormales $(\%)$.

Tabla 2 Características macroscópicas y microscópicas de semen fresco de llama (Lama glama) sin la adición de dilutores, colectado por el método de electroeyaculación

\begin{tabular}{|c|c|c|c|c|c|}
\hline \multirow{2}{*}{ Características macroscópicas } & \multirow{2}{*}{ Promedio } & \multirow{2}{*}{ SD \pm} & \multirow{2}{*}{ C.V. $(\%)$} & \multicolumn{2}{|c|}{ Extremos } \\
\hline & & & & Máx. & Mín. \\
\hline Color & Blanco translucido & - & - & - & - \\
\hline Volumen (mL) & 1.67 & 0.29 & 17.32 & 2 & 1.5 \\
\hline Características microscópicas & Promedio & SD \pm & C.V. $(\%)$ & Máx. & Mín. \\
\hline Concentración de Espermatozoides (millones/mL) & 39.14 & 11.22 & 57.38 & 51.2 & 29 \\
\hline Motilidad espermática (\%) & 22.8 & 5.6 & 24.56 & 28.4 & 17.2 \\
\hline Vitalidad de Espermatozoides. (\%) & 86.67 & 5.77 & 6.66 & 90 & 80 \\
\hline
\end{tabular}

$\mathrm{SD} \pm=$ Desviación estándar; C.V. = Coeficiente de variación 
Tabla 3 Resultados del ANVA y estadísticos generales para la motilidad espermática (\%) al post-descongelamiento

\begin{tabular}{|c|c|}
\hline Factores principales & Motilidad espermática (\%) \\
\hline Dilutor & $0,004 *$ \\
\hline Bloque & $0,500 \mathrm{NS}$ \\
\hline \multicolumn{2}{|c|}{ Estadísticos generales } \\
\hline $\mathrm{N}$ & 15 \\
\hline $\mathrm{X}$ & 8.52 \\
\hline C.V. $(\%)$ & 15.23 \\
\hline Extremos (Máx. - Mín.) & $19.1-0$ \\
\hline
\end{tabular}

* = Significativo $(\mathrm{P}<0,05), \mathrm{NS}=$ No significativo $(\mathrm{P}>0,05), \mathrm{n}=$ número de observaciones

$\mathrm{x}=$ Promedio general, $\mathrm{C} . \mathrm{V} .=$ Coeficiente de variación .

Tabla 4 Comparación de medias para el factor dilutor en la variable motilidad espermática (\%) al post-descongelamiento

\begin{tabular}{lcc}
\hline \multicolumn{1}{c}{ Dilutor } & $\begin{array}{c}\text { Promedio } \\
\text { Motilidad } \\
(\%)\end{array}$ & Duncan $(\boldsymbol{\alpha}=\mathbf{0 . 0 5})$ \\
\hline $\begin{array}{l}\text { Tris-ácido cítrico-fructosa-yema- } \\
\text { glicerol } \\
\text { AndroMed }\end{array}$ & 17.03 & $\mathrm{~A}$ \\
\hline
\end{tabular}

Tabla 5 Resultados del ANVA y estadísticos generales para el porcentaje de vitalidad

\begin{tabular}{cc}
\hline Factores principales & Vitalidad espermática (\%) \\
\hline Dilutor & $0.004^{*}$ \\
Bloque & $0.330 \mathrm{NS}$ \\
\hline \multicolumn{2}{c}{ Estadísticos generales } \\
\hline $\mathrm{n}$ & 15 \\
C.V. (\%) & 11.97 \\
Extremos (Máx. - Mín.) & 10.1 \\
\hline
\end{tabular}

$*$ Significativo $(\mathrm{P}<0,05), \mathrm{NS}=$ No significativo $(\mathrm{P}>0,05), \mathrm{n}=$ número de observaciones,

$\mathrm{x}=$ Promedio general, C.V. $=$ Coeficiente de variación 
Figura 2 Comparación de medias Duncan para el factor dilutor en el porcentaje de vitalidad espermática

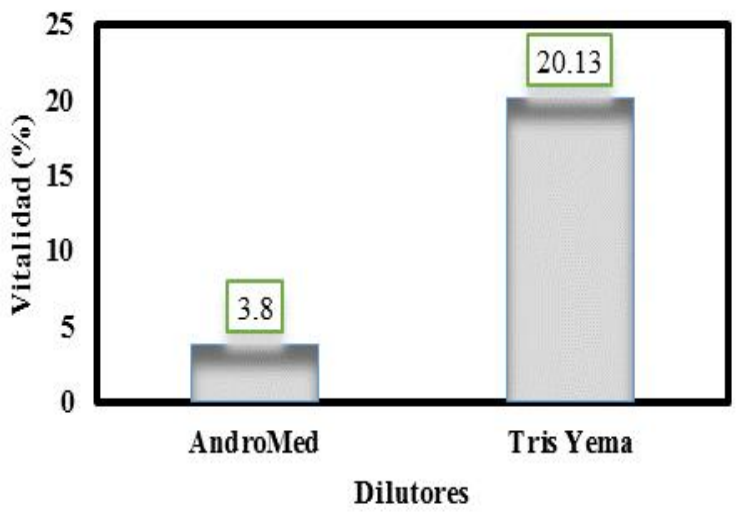

Tabla 6 Resultados del ANVA y estadísticos generales para el porcentaje de espermatozoides anormales

\begin{tabular}{lc}
\hline \multicolumn{1}{c}{ Factores principales } & Anormalidades (\%) \\
\hline \hline Dilutor & $0.018^{*}$ \\
Bloque & $0.188 \mathrm{NS}$ \\
\hline \hline \multicolumn{2}{c}{ Estadísticos generales } \\
\hline $\mathrm{n}$ & 15 \\
$\mathrm{x}$ & 9.28 \\
$\mathrm{C} . \mathrm{V} .(\%)$ & 3.16 \\
Extremos (Máx. - Mín.) & $10.36-7,73$ \\
\hline
\end{tabular}

$*$ Significativo $(\mathrm{P}<0,05), \mathrm{NS}=$ No significativo $(\mathrm{P}>0,05)$, $\mathrm{n}=$ número de observaciones, $\mathrm{x}=$ Promedio general, C.V. = Coeficiente de variación

Figura 3 Comparación de medias para el factor dilutor en el porcentaje de espermatozoides anormales

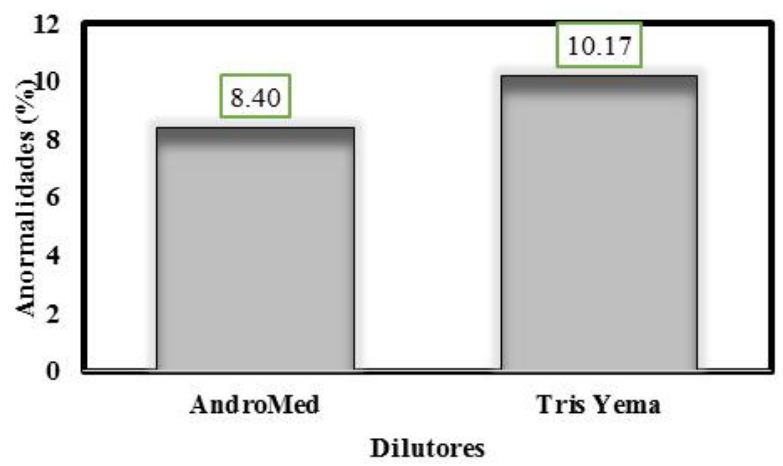

\section{Discusión}

El semen de llama colectado por el método de electroeyaculación (EE), presentó un volumen promedio de $1.67 \pm 0.29 \mathrm{~mL}$, color blanco lechoso, concentración espermática de $39.14 \times 10^{6}$ espermatozoides/mL, motilidad espermática de $22.80 \%$ y una vitalidad de $86.67 \%$. Tabla 1 .

El color de semen obtenido por el método de electroeyaculación (blanco translucido), es idéntico al reportado por otros autores Cruz \& DelgadoCallisaya 2011, Valle 2013). El color del semen indica la concentración de espermatozoides (Bustinza 2001).

El volumen de semen colectado fue de $1.5-2 \mathrm{~mL}$, similar al descrito por Giuliano et al. (2008), Cruz \& Delgado (2011), quienes obtuvieron $1.61 \mathrm{~mL}$ y $1.4 \mathrm{~mL}$ respectivamente, colectados EE. La concentración espermática promedio en nuestra investigación fue de $39.14 \times 10^{6}$ espermatozoides $/ \mathrm{mL}$, similar al reportado por Giuliano et al. (2008), Cruz \& Delgado-Callisaya (2011) quienes obtuvieron concentraciones promedios de $33.01 \times 10^{6}$ y $29.00 \times 10^{6}$ espermatozoides $/ \mathrm{mL}$ respectivamente.

Valle (2013), evaluando dos técnicas de colección en llamas (Lama glama) en la estación Experimental de Choquenaira, obtuvo volúmenes de $1.56 \mathrm{~mL}$ con vagina artificial (AV) y $0.25 \mathrm{~mL}$ con $\mathrm{EE}$, quien atribuye estos resultados al método de colecta, indicando que con el método de vagina artificial se obtiene mayores volúmenes que con el de EE.

La misma autora indica, que el bajo volumen por el método de EE, podría deberse a que no hubo una excitación previa por presencia de una hembra, el eyaculado obtenido solo pudo provenir del testículo y no de las glándulas bulbo uretrales ni del cuerpo de la próstata, las cuáles son dependientes de ciertas hormonas (FSH, LH y TT). La colección simplemente pudo haber sido por las descargas eléctricas 
que solo obligo al testículo a que este libere su contenido.

Chiri (2002), indica que el volumen es muy variable, ya que la cantidad de eyaculado dependerá no solo de la edad, sino del estado fisiológico, al igual que de aquellos factores exotérmicos y alimenticios, los cuales son relevantes para la obtención de un buen volumen de eyaculado, reportando volúmenes superiores de aquellos machos jóvenes que inician la vida sexual a partir de los dos años de edad, el cuál es un eyaculado rico espermáticamente con alta viabilidad en épocas húmedas.

La concentración espermática promedio en nuestra investigación fue de $39.14 \times 10^{6}$ espermatozoi$\mathrm{des} / \mathrm{mL}$, similar al reportado por otros autores como Giuliano et al. (2008), Cruz (2011) quienes obtuvieron concentraciones promedios de $33.01 \times 10^{6}$ y $29.00 \times 10^{6}$ espermatozoides $/ \mathrm{mL}$ respectivamente, colectados por el mismo método EE. Este color es un indicador de la concentración obtenida en este trabajo.

La motilidad espermática en el semen colectado fue $22.80 \%$, similar al obtenido por Giuliano et al. (2008) quienes reportaron una motilidad progresiva menor a 20\%, Enciso (2009), Valle (2013) obtuvieron valores superiores al conseguido en esta investigación: $28 \%, 46.08 \%$ y $53.95 \%$ respectivamente, todos colectados por el método de EE. El plasma seminal, es altamente viscoso (parecido a la clara de huevo), lo que dificulta la motilidad masal de los espermatozoides presentando tan solo una motilidad progresiva después de la aplicación del dilutor y licuefacción.

La vitalidad promedio fue del $86.67 \%$ superior a los resultados obtenidos por Cruz (2011), Valle (2013), quienes reportaron promedios de $67.25 \%$ y $60.83 \%$ respectivamente, autores como Enciso (2009), Garabito (2003), sugieren tomar en cuenta al diluyente empleado para proteger a los espermatozoides de factores externos como el cambio de temperatura. Este hecho fue estrictamente seguido en la metodología de dilución por lo que posiblemente se obtuvo este valor tan alto.

Motilidad espermática post descongelación de semen de llama crioconservado en dos dilutores (AndroMed y Tris-ácido cítrico-fructosa-yema-glicerol)

De acuerdo al ANVA hay diferencias significativas entre dilutores $(\mathrm{P}<0.05)$ y no así entre bloques $(\mathrm{P}>0.05)$. Tabla 3. El coeficiente de variación fue de $15.23 \%$. Mediante la prueba de Duncan $(\alpha=0.05)$, para el factor dilutor, se obtuvo dos rangos. En el primer rango (A), se encuentra el dilutor tris ácido cítrico-fructosa-yema-glicerol, con un promedio de $17.03 \%$ de motilidad espermática y en el segundo rango (B) el dilutor AndroMed, con un promedio de $0 \%$.

De acuerdo con el Tabla 4, podemos establecer que las diferencias que existen entre ambos dilutores, se deben a que el dilutor AndroMed no presenta yema de huevo en su composición, pues esta preserva mejor el semen durante el proceso de refrigeración Salomón \& Maxwell (2000), que protege a los espermatozoides contra el choque al frío, protegiéndolos durante el congelamiento y descongelamiento.

Giuliano et al. (2008), Bravo et al. (2002), indican que los diluyentes que contienen lactosa-yema de huevo y tris-ácido cítrico resultarían ser mejores para proteger a los espermatozoides de camélidos.

En cuanto al promedio general se obtuvo un valor de $8.52 \%$ de motilidad espermática al postdescongelamiento, valor que descendió desde la colecta $(22.8 \%)$ al post-descongelamiento, a una razón de $14.28 \%$, este resultado se atribuye al tiempo de criopreservación del semen, es decir a medida que pasa el tiempo la calidad seminal disminuye Delgado-Calisaya et al. (2003) que obtuvieron un promedio de $12.03 \%$ evaluando el efecto de tres 
dilutores para la congelación del eyaculado de llama (Lama glama). Tabla 3.

Al respecto Aller et al. (2003), evaluando la influencia de la criopreservación sobre la motilidad, viabilidad y fertilidad de espermatozoides de llama (Lama glama) observaron que el porcentaje promedio y desviación estándar de espermatozoides motiles disminuyó significativamente $(\mathrm{P}<0.05)$ de 54.3 \pm 10.5 en muestras de semen fresco a $20.4 \pm 7.5$ en muestras congeladas/descongeladas. Aisen et al. (2012), probando el efecto de diferentes concentraciones de trealosa en la congelación de semen de llama obtuvo un promedio general de $4.74 \%$ de motilidad espermática

Una de las principales características de los espermatozoides criopreservados es la disminución de la proporción de células móviles a valores entre 40 a $50 \%$ (Watson 2000)

Vitalidad espermática post descongelación de semen de llama crioconservado en dos dilutores (AndroMed y Tris-ácido cítrico-fructosa-yema-glicerol) Los resultados obtenidos en el análisis de varianza del porcentaje de espermatozoides vivos, nos muestran que existen diferencias significativas entre dilutores $(\mathrm{P}<0.05)$ y no así entre bloques $(\mathrm{P}>0.05)$, (tabla 5) con un coeficiente de variación de $28 \%$. Al encontrar diferencias significativas $(\mathrm{P}<0.05)$ entre dilutores, se realizó la comparación de medias, mediante la prueba de Duncan a un nivel de 5\%, donde se aprecia que el dilutor tris-ácido cítrico-fructosayema-glicerol presenta el mayor porcentaje de vitalidad (20.13\%) a comparación del dilutor AndroMed (3.80\%). Figura 2

El promedio general al post-descongelamiento refleja un valor de $11.97 \%$ de vitalidad, valor que descendió desde la colecta (88.67\%) al post descongelamiento, a una razón de $76.7 \%$, esta variación se podría deber al cambio de temperatura, estrés osmótico, formación de hielo intracelular. Al respecto 17
Salomón (2011), indica que la baja viabilidad se debe a factores como: cambio de temperatura, estrés osmótico, formación de hielo intracelular y toxicidad.

El cambio de temperatura produce un estrés en la membrana, posiblemente relacionado con un cambio de fase en los lípidos. Las lesiones producidas por el congelamiento de las membranas antes y durante el congelamiento sólo se invierten parcialmente después del descongelamiento. Finalmente la toxicidad de algunas sustancias como el glicerol también produce la muerte de los espermatozoides. Santalla (2013), evaluando el efecto de cuatro dilutores sobre la viabilidad espermática de llama ( $L a$ ma glama), reporto que el dilutor tris-yema-glucosa mantuvo una mayor vitalidad de los espermatozoides, ya que este dilutor contenía como fuente energética a la glucosa, el cuál es utilizado por los espermatozoides para la producción de ATP. Delgado-Callisaya et al. (2003), Aisen et al. (2012), reportaron valores promedios de vitalidad de $13.78 \%$ y $4.74 \%$ respectivamente.

Gonzáles et al. (2008), evaluando el efecto de dilutores en la vitalidad de los espermatozoides de semen de alpacas, obtuvo los siguientes resultados: T1 tris-fructosa-yema de huevo $(78.4 \pm 3.8 \%)$, T2. citrato-glucosa-yema de huevo $(75.2 \pm 6.5 \%)$, T3 citrato glucosa-yema-tripsina $(81.5 \pm 4.2 \%)$, T4 citrato glucosa-yema colagenasa $(79.6 \pm 5.3 \%)$, T5 albúmina sérica bovina $(\mathrm{BSA})+$ glucosa $(67,5 \pm 6,4 \%)$ y $\mathrm{T} 6$. BSA+glucosa+efecto mecánico $(65.3 \pm 7.2 \%)$, donde los valores para T3, T4 y T1 son mayores $(\mathrm{P}<0.01)$ a los de T5. Estos resultados sugieren que los dilutores utilizados responden con eficiencia a un manejo de semen fresco práctico, de bajo costo y de fácil provisión.

Aller et al. (2003), evaluando la influencia de la criopreservación sobre la motilidad y viabilidad de espermatozoides de llama (Lama glama) observaron 
que el porcentaje promedio y desviación estándar de espermatozoides viables disminuyó significativamente $(\mathrm{P}<0.05)$ de $68.5 \pm 12.3$ en muestras de semen fresco a $32.4 \pm 10.5$ en muestras congeladas/descongeladas.

Porcentaje de espermatozoides anormales en semen de llama crioconservados en dos dilutores (AndroMed y Tris-ácido cítrico-fructosa-yema-glicerol) Los resultados del tabla 6, para esta variable muestran que existen diferencias significativas entre dilutores $(\mathrm{P}<0.05)$ y no así entre bloques $(\mathrm{P}>0.05)$.

El coeficiente de variación fue de $3.16 \%$, que indica que los datos obtenidos son confiables y la variabilidad de las unidades experimentales fue mínima dentro de cada tratamiento (menores a 30\%) (Ochoa 2007).

Realizando la prueba de Duncan a un nivel de significancia $\alpha=0.05$, para el factor dilutor (Figura 3), se obtuvo diferencias significativas $(\mathrm{P}<0.05)$, por lo que se distinguen dos rangos. En el primer rango (A), se encuentra el dilutor tris-ácido cítricofructosa-yema-glicerol, con un promedio de $10.17 \%$ de espermatozoides anormales y en el segundo rango (B) el dilutor AndroMed, con un promedio de $8.40 \%$ de espermatozoides anormales. Ambos valores resultaron ser similares a los reportados en semen fresco comparado con otras investigaciones.

Comparación de medias para el factor dilutor en el porcentaje de espermatozoides anormales. La figura 3 , refleja el mayor porcentaje de células espermáticas anormales (\%) con el dilutor tris-ácido cítricofructosa-yema-glicerol, con un porcentaje de $10.17 \%$ el cual difiere y supera con $1.77 \%$ al dilutor AndroMed con un promedio de $8.40 \%$, estos resultados probablemente se atribuyen a la forma de colección del semen (EE), ya que ocasiono estrés en las llamas en el momento de la colecta, sin embargo el porcentaje de espermatozoides anormales se encuentran dentro del rango permisible, entre las anormalidades más frecuentes que se encontraron, fueron las siguientes: defectos de pieza media, decapitados, colas sueltas, colas dobladas y bicéfalos.

Los resultados obtenidos son corroborados por Bearden \& Fuquay (1995), quienes indican, que en cada eyaculación habrá espermatozoides anormales. El límite permitido es de 8 a $10 \%$ los que no tienen efectos adversos sobre la fertilidad. Si los espermatozoides anormales son más de $25 \%$ del total eyaculado, se puede anticipar una reducción de la fertilidad.

Generalmente las alteraciones en la calidad espermática están relacionadas a tres grandes factores: genéticos, nutricionales y ambientales. Por ejemplo, Vaughan (2002), en estudios realizados en Australia, menciona que es común el hallazgo de elevados índices de espermatozoides anormales en CSA (Camélidos Sudamericanos) domésticos, dato relacionado a la baja tasa de fertilidad que tienen éstas especies.

Por otra parte Giuliano et al. (2008), encontraron valores de $16.58 \pm 8.45 \%$ de espermatozoides anormales.

Al respecto Valle (2013), encontró $7.92 \%$ de espermatozoides anormales con el método de electroeyaculación, entre ellos están: macrocéfalos, bicéfalos, microcéfalos, decapitados, gota citoplasmática proximal, gota citoplasmática distal, colas torcidas y colas enrolladas.

Las características fisiológicas reproductivas de los camélidos sudamericanos dificultan los intentos de colectar semen de una forma segura, para que pueda hacerse rutinaria, su colección de semen aún no se encuentra investigada del todo, diferentes centros de investigación en camélidos sudamericanos no establecen acuerdos de que técnica es apropiada, y así unificar criterios para resolver este problema en su reproducción. 
Las características del semen fresco de llamas colectadas por la técnica de electroeyaculación son diferentes a los obtenidos en otras especies de camélidos sudamericanos debido a la variabilidad existente entre especies. Además se determinó que el mayor tiempo de refrigeración afecta negativamente al porcentaje de motilidad de las muestras refrigeradas.

Por lo anteriormente mencionado, en los CSA la presencia del PS no permite un buen manejo del semen, dificultando la interacción de los espermatozoides con crioprotectores y el envasado de las pajuelas, por lo tanto se hace necesario un tratamiento enzimático que posibilite el manejo de las muestras a criopreservar. Por otra parte, la dilución o tratamiento enzimático de los eyaculados podría modificar el patrón de movilidad de los espermatozoides, interferir en la interacción oviducto-espermatozoide y favorecer fenómenos de membrana similares a la capacitación, comprometiendo el encuentro de los gametos en el oviducto. Teniendo en cuenta que hasta la actualidad no hay un protocolo de criopreservación de semen de CSA que permita obtener preñez, es de interés realizar más estudios para conocer los efectos o influencia de la dilución del plasma seminal en la fisiología y criopreservación del espermatozoide de llama

La conservación y el mantenimiento de la vitalidad de los espermatozoides con ayuda de dilutores, ayudo a mejorar enormemente los porcentajes de conservación con relacion a los diferentes trabajos de investigación realizados a la fecha.

\section{Conflicto de interés}

El presente trabajo ha cumplido con normas éticas en el manejo de los animales y no genera conflictos de interés.

\section{Agradecimientos}

Los autores agradecen al Instituto de Investigación en Ciencia Animal y Tecnología (IICAT), Medicina Veterinaria y Zootecnia. Laboratorio de Terinología.

A los técnicos de Palcoco por brindarnos los animales para esta investigación.

\section{Literatura citada}

Agraz GA. Caprinotecnia 2. Gestación. Primera Edi ción. Editorial LIMUSA, México, DF. 1989. p 1666.

Aisen EG, Bérgamo NS, Medina VH, Martínez CY, Vagnoni YC. Efecto de la trehalosa en la congelación de semen de llama. Parámetros de integridad espermática in vitro. IV Congreso Mundial de Camelidos Sudamericanos. Arica. Chile. 2012. p 118.

Aller JF, Rebuffi GE, Cancino AK, Alberio RH. Influencia de la criopreservación sobre la motilidad, viabilidad y fertilidad de espermatozoides de llama (Lama glama). Arch Zootec. 2003; 52: 15-23.

Bearden JH, Fuquay JW. Reproducción Animal Aplicada. 4ta edición, Ed. El Manual Moderno. México, D.F.1995; p. 220.

Bravo F. Estudio comparativo de tres métodos de colección de semen en alpacas. Tesis de Bachiller. Facultad de Zootecnia, Univ. Nacional Agraria La Molina. Lima. 1989. p. 96.

Bravo PW, Flores U, Garnica J, Ordoñez C. Collection of semen and artificial insemination of alpacas. Theriogenology. 1997a; 47: 619-626.

Bravo PW, Moscoso R, Alarcón V, Ordóñez C. Ejaculatory process In: Johnson LW (eds) Update on Llama Medicine. Veterinary Clinics of 
North America Food Animal Practice WB Saunders, Philadelphia. 2002. p. 100-132.

Bustinza V. La alpaca. p 158-184. Oficina de Recursos de Aprendizaje. Perú. 2001.

Cárdenas O, Huanca T, Sapana R, Alarcón V. Avances de inseminación artificial de llamas con semen congelado. XXII Reunión Científica Anual de la Asociación Peruana de Producción Animal. Huancavelica-Perú. 1999.

Chiri R. Producción de Camélidos Sudamericanos. Oruro - Bolivia. 2002.

Cruz A, Delgado-Callisaya P. Desarrollo de un electroeyaculador para llamas (Lama glama) en el Departamento de La Paz. [tesis licenciatura]. Zootecnia-Unidad Académica Campesina Tiahua nacu. La Paz, Bolivia. 2011.

Delgado-Callisaya P, Flores F, Fernández R, González V, Maceda E, Copa S, Medina J. Técnicas de colección de semen en llamas. III Congreso mundial de camélidos. Potosí Bolivia. 2003.

Delgado-Callisaya PA. 2010. Apuntes de maestría. Arkansas of University. Arkansas - USA.

Enciso M. Reproducción en la vicuña macho Vicugna vicugna: evaluación del método de contención química, colección de semen, análisis del eyaculado y biometría testicular. [tesis licenciatura]. Lima Universidad Nacional Mayor de San Marcos. Perú. 2009.

Evans G, Maxwell WMC. Salamon's Artificial Insemination of Sheep and Goats. Buterworths Pty. Limited, Sydney. Australia. 1987.

Fernández-Baca S, Calderón W. Métodos de colección de semen de la alpaca. Rev Fac Med Vet Perú. 1965; 18-19-20: 13-17.

Ferré L, Werkmeister A. Desarrollo de una vagina artificial termoeléctrica para la colecta de semen. Rev Agr Prod Anim. 1996; 16: 363-365.

Garabito A. Evaluación física de semen en llama (Lama glama) q'hara y tampulli. [tesis licencia- tura] Oruro. Universidad Técnica de Oruro. Bolivia. 2003: p. 90-93.

Garnica J, Achata R. Constituyentes químicos del plasma seminal de la alpaca. Resúmenes XII Reunión. Asoc Per Prod Anim. Lima. 1998. p 66.

Giuliano S, Director A, Gambarotta M, Trasorras V, Miragaya M. Collection method, season and individual variation on seminal characteristics in the llama (Lama glama). Anim Reprod Sci. 2008; 104: 359-369.

Giuliano S, Director A, Gambarotta M, Trasorras V, Miragaya M. Influencia de diluyentes en semen refrigerado a $5{ }^{\circ} \mathrm{C}$ durante 24 horas en la especie Lama glama. Programa Nacional Investigación Camélidos - INIA - Puno - Perú. Facultad de Medicina Veterinaria - UNMSM- Lima Perú. IV Congreso Mundial sobre Camélidos (Santa María, Provincia de Catamarca. República Argentina, 2006).

Gonzales H, Dávalos R, Moina M, Mellisho E. Obtención y criopreservación de espermatozoides de alpacas. Scientia. 2008: 10: 223-234.

Hafez E. Reproducción e Inseminación Artificial en Rumiantes, $6^{a}$ ed. en castellano. Edit. McGrawHill; 1996; p 300-330.

Herrera E. Evaluación de dilutores para la conservación de semen en ovinos. Tesis de Bachiller. Facultad de Medicina Veterinaria y Zootecnia, Univ. Nacional del Altiplano. Puno. 1986. p 72.

IGM (Instituto Geográfico Militar). Mapa de localización, Estación Experimental de Kallutaca, La Paz, Bolivia. 2005.

López J. Evaluación de la morfología espermática del semen de camélidos sudamericanos tratado con tripsina. III Congreso mundial de camélidos. Potosí. 2001.

Marín JC, Spotorno AE, González BA, Bonacic C, Wheeler JC, Casey CS, Bruford MW, Palma RE, 
Poulin E. Mitochondrial DNA variation and systematics of the guanaco (Lama Guanicoe. Artiodactyla: Camelidae). Journal of Mammalogy. 2008; 89: 269-281.

Mendoza O. Determinación de la sobrevivencia de los espermatozoides de alpaca (Lama pacos) colectados del conducto deferente con el uso de tres dilutores. [tesis licenciatura] Puno. Perú. 2001.

Mogrovejo D. Estudios de semen de la alpaca. [tesis bachiller]. Lima: Facultad de Medicina Veterinaria, Universidad Nacional Mayor de San Marcos. 1952. p 21.

Ochoa T. Diseños experimentales. Universidad Mayor de San Andrés Facultad de Agronomía, La Paz. Bolivia. 2007.

Ratto M, Wolter M, Gómez C, Berland M. Refrigeration of epididymal sperm from lama with three different extenders. En: II Congreso Mundial sobre Camélidos. Cusco, Perú. 1999.

Ruiz JA. Comercialización de productos y subproductos de la explotación de camélidos sudamericanos. Resumen de conferencia. IV Congreso Latinoamericano de Especialistas en Pequeños Rumiantes y Camélidos Sudamericanos. Curitiba-Brasil. 2005.

Salomón A. Capacitación del espermatozoide caprino durante el proceso de estabilización pre crioconservación. Veracruz-México 2011.
Salomon S, Maxwell WMC. Storage of ram semen, Anim Reprod Sci. 2000; 62: 77-111.

Santalla IS. Viabilidad espermática del semen de llama (Lama glama) refrigerado en cuatro dilutores. [tesis licenciatura]. La Paz. Zootecnia. Unidad Académica Campesina Tiahuanacu. La Paz: Bolivia. 2013.

Sumar J, Leyva V. 1981. Colección de semen mediante la vagina artificial en alpaca. En: Resúmenes IV Conf. Internacional en Camélidos Sudamericanos. Punta Arenas, Chile. 1981.

Sumar J. Avances y perspectivas en reproducción de camélidos. I Symposium Internacional Avances en Reproducción de Rumiantes. Lima. 1997. p 30.

Torres H. South American Camelids. An action plan for their vonservation. (Editor). IUCN/SSC South American Camelid Specialist Group. Gland, Suiza. 1992.

Valle EM. Evaluación de dos técnicas de colección de semen en llamas (Lama glama) en la Estación Experimental de Choquenaira. [tesis licenciatura]. La Paz. Universidad Mayor de San Andrés. Bolivia. 2013. p. 162.

Vaughan J. Improving the efficiency of reproduction and breeding in alpacas. Rural Industries Research and Development Corporation, Barton. Australia. 2003. p. 20.

Watson P. The causes of reduced fertility with cryopreserved semen. Anim Repro Sci. 2000; 6061: 481-492. 